Conclusions It was possible to obtain reliable data under physiological conditions using Fecobionics. Five defecatory phases could be defined by the pressure signature. Orientation and bending could also be assessed.

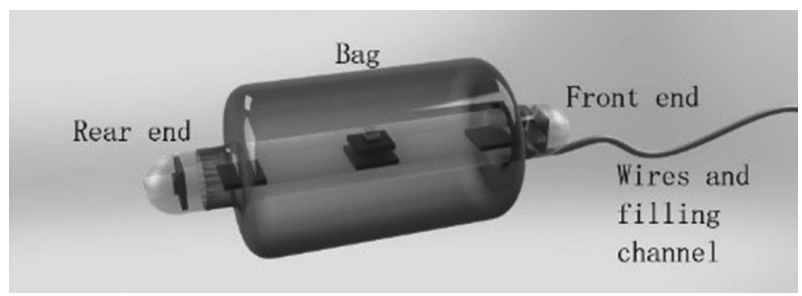

Abstract IDDF2018-ABS-0174 Figure 1

\section{IDDF2018-ABS-0175 FUNCTIONAL LUMEN IMAGING PROBE ASSESSMENT OF ANAL CANAL DISTENSIBILITY}

Ssu Chi Chen*, Kaori Futaba, Wing Wa Leung, Cherry Wong, Tony Mak, Simon Ng, Hans Gregersen. Department of Surgery, The Chinese University of Hong Kong, Hong Kong

\subsection{6/gutjnl-2018-IDDFabstracts. 141}

Background The mechanism of defaecation and continence is a complex process involving several factors. Identifying the cause of faecal incontinence is often difficult. Assessment of patients currently involve functional assessment with highresolution anorectal manometry (HR-ARM) and anatomical assessment using Endoanal ultrasound (EAUS) scan, providing limited information. EndoFLIP (Functional Lumen Imaging Probe) allows additional assessment of anal canal distensibility. 12

Aim To compare anal canal function using EndoFLIP between faecally incontinent patients (FI) and asymptomatic subjects (AS).

Methods All subjects were assessed using HR-ARM, EAUS and EndoFLIP. Using the EndoFLIP, continuous ramp distension was carried out up to $5=$ bag vol. In second ramp distension, the $5=$ volume was maintained while the subjects were asked to squeeze their anus. Anal competence of the narrowest area of the anal canal was evaluated using three distinct parameters derived from the EndoFLIP data. Mann-Whitney's U test was used for statistical analysis.

Results Sixteen FI patients (2M/14F, Mean age 61.38 years, SEM +3.34) and 9 AS (3M/6F, Mean age 57.9 years, SEM +3.69 ) were assessed. The median yield pressure (the pressure when the diameter increased from baseline) was significantly lower in the FI group at $22.99 \mathrm{cmH}_{2} \mathrm{O}\left(17.67-45.49 \mathrm{cmH}_{2} 0\right)$ compared to $55.95 \mathrm{cmH}_{2} \mathrm{O}\left(36.56-64.82 \mathrm{cmH}_{2} 0\right)$ in asymptomatic subjects. There was no significant difference in the distensibility between the FI group $0.18(0.17-0.35) \mathrm{mm} / \mathrm{cmH}_{2} 0$ compared to AS group $0.23(0.12-0.39) \mathrm{mm} / \mathrm{cmH}_{2} \mathrm{O}$ calculated as the change in diameter divided by the change in distension pressure. The squeeze strength was significantly higher in the AS group $167.3(62.0-270.8) \mathrm{mm} . \mathrm{cmH}_{2} \mathrm{O}$ compared to FI group $23.6(8.1-86.1) \mathrm{mm}_{\mathrm{cmH}} \mathrm{c}$.

Conclusions EndoFLIP demonstrated that FI patient's anal sphincters yielded at a lower pressure and had lower squeeze strength than in asymptomatic subjects, which may be clinically more relevant than squeeze pressures alone.

\section{IDDF2018-ABS-0177 THE NEW INVESTIGATION OF THE NURSING MEASURES ON ENDOSCOPIC ULTRASONOGRAPHY (EUS) FOR DEEP GASTRIC BIOPSY}

Rong Pei ${ }^{\star}$, Xiuliang Yang. Shanghai Cancer Center Hospital, China

\subsection{6/gutjnl-2018-IDDFabstracts. 142}

Background To investigate the effect of the nursing measures on endoscopic ultrasonography (EUS) for deep gastric biopsy, in order to improve the success rate and safety of biopsy.

Methods 48 patients that have underwent ordinary gastroscopy more than once. At foreign hospital and the pathological results are negative, underwent endoscopic ultrasonography deep biopsy for diagnosis in our hospital. We retrospectively analysis and summarise the importance of the preoperative perfect preparation, the high quality of nursing in operation, postoperative close observation and nursing, and every step in operation.

Results 48 cases were diagnosed by endoscopic ultrasonography. The procedure was smooth in 46 cases, 2 patients reacted with nausea and coughing. After examination, 19 patients complained of pharynx discomfort, and 1 cases had a small amount of bleeding after the operation and recovered after treatment.

Conclusions Focusing on every step and details and high-quality nursing can effectively relieve pain and improve patient compliance during ultrasonic endoscopic gastric for deep biopsy; Effective coordination of medical and nursing care can improve the positive diagnosis rate and safety of biopsy. Ensuring successful operation.

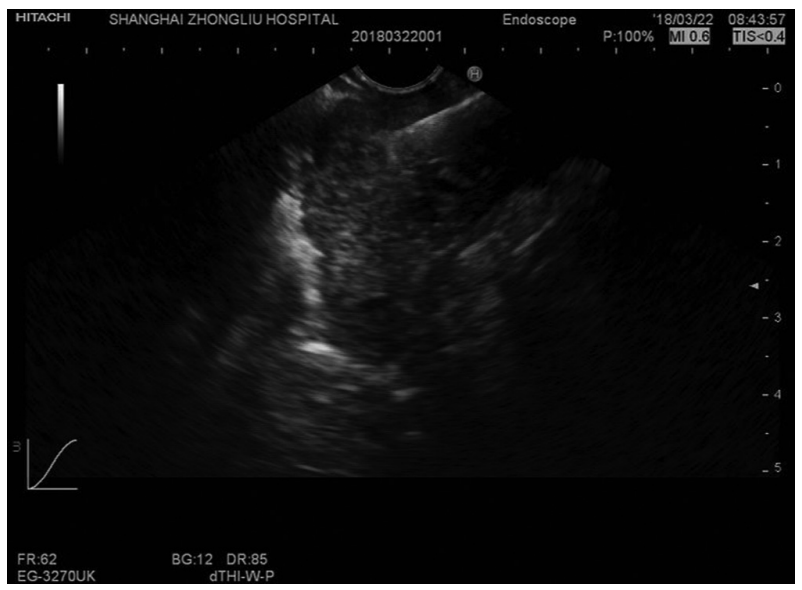

Abstract IDDF2018-ABS-0177 Figure 1

\section{IDDF2018-ABS-0179 AZATHIOPRINE: WHERE IT STANDS IN ACHIEVING NEWER MUCOSAL HEALING GOAL IN ULCERATIVE COLITIS: A COHORT STUDY}

${ }^{1}$ Rohan Badave*, ${ }^{2}$ BV Tantry, ${ }^{3}$ Sandeep Gopal, ${ }^{3}$ Suresh Shenoy. 'Senior Resident, Department of Gastroenterology, Kasturba Medical College, Mangalore, Karnataka, India; ${ }^{2}$ Professor, Department of Gastroenterology, Kasturba Medical College Mangalore, Karnataka, India; ${ }^{3}$ Associate Professor, Department of Gastroenterology, Kasturba Medical College, Mangalore, India

10.1136/gutjnl-2018-IDDFabstracts. 143 